\title{
A computational approach to investigation of $C$. elegans backwards crawling mechanism via simulation of involved nervous and muscular cells activity driving body movement
}

\author{
Andrey Yu. Palyanov \\ A.P. Ershov Insitute of Informatics Systems, Novosibirsk, Russia \\ Novosibirsk State University, Novosibirsk, Russia \\ palyanov@iiis.nsk.su
}

\author{
Natalia V. Palyanova, \\ Institute of Molecular Biology and Biophysics, \\ Novosibirsk, Russia, \\ natasha@soramn.ru
}

\begin{abstract}
A small and well-studied invertebrate, C. elegans, is often considered to become the first organism reproduced in the form of a computer simulation. However, its nervous system, with hundreds of different ion channels and thousand of connections between neurons remains too sophisticated for a complete reconstruction nowadays. The subject of our research is a small subsystem including few dozens of neurons, body wall muscle cells, proprioceptors and interactions between them, which was proved to be sufficient for producing backwards crawling independently of the rest nervous system. We present the models of all key components and interactions between them, as well as the software system for performing simulations.
\end{abstract}

Keywords - C. elegans, computational modeling, backwards crawling system, biomechanics, neurobiology, rhythmic activity, proprioceptive feedback

Motivation and aim

Motivation

In computational neurobiology it is highly important to know whether the model of neural activity is able to produce the same dynamics in the same situation in comparison with the real organism. For $C$. elegans, even in case when it is freely moving at natural conditions, it is possible to observe the time dependence of its individual neurons activity [1]. The system responsible for backwards crawling is an outstanding object for modeling and tuning of the model via comparison with the real experimental data.

Aim

The purpose of this investigation is to build a detailed biologically reasonable simulation of the described system, composed of the nervous and muscular cells with quite wellknown properties and structure of the interconnections, but without the knowledge about how all these functions together, taking into account proprioception and feedback from the virtual physical environment. The second part to explore the cabability of simulated system to produce the expected behavior - backwards crawling - via optimization of the model's electrophysiological parameters keeping them within biologically reasonable intervals of values.

\section{Methods}

3D physical body of C. elegans and biomechanics of its locomotion is simulated using our software system named Sibernetic [2], which is able to calculate the dynamics of liquid, elastic matter, contractile matter (for muscle fibers simulation) and elastic films (optionally impermeable to liquid). Sibernetic is able to function together with the NEURON simulation environment, aimed at biological neurons and neural networks modeling, performing data exchange to send sensory information from physical body to nervous system and activating signals from electrophysiological representation of motorneurons to their biomechanical model.

C. elegans neurons differ from those of "more advanced" biological species which have spiking neurons interacting via action potentials - there is evidence that signals travelling through $C$. elegans nervous system propagate passively, in a gradual manner [3]. Previously we have created the basic model of the $C$. elegans neuron (within the NEURON simulator), with typical morphological properties and electrophysiological parameters responsible for passive signal propagation [4], defining its speed and length constant. However, there is an experimental evidence that some of $C$. elegans neurons can function as non-bursting oscillators - like A-class motorneurons, exhibiting intrinsic rhythmic activity at frequencies less than $1 \mathrm{~Hz}$ (without external stimulation) [5]. In contrast to C. elegans neurons, its body wall muscles are able to fire action potentials [6], even spontaneously.

The main types of interactions between the mentioned cells are the following:

1) Chemical synapses and gap junctions between neurons

2) Graded transmission at the neuromuscular junctions of C. elegans, encoded by post-synaptic current bursts [7] more frequent action potentials cause larger degree of muscle contraction and vice versa

3) Spatially adjacent body wall muscles are connected via gap junctions, which, at some degree, synchronize action potentials and $\mathrm{Ca}^{2+}$ transients in them [8].

4) A-class motorneurons, which were active while backward locomotion, possess long undifferentiated processes, oriented anteriorly regarding to neuron's soma positions, which hypothetically function as stretch receptors [9].

\section{Results}

In addition to Sibernetic and its interaction with the NEURON, we've developed a program, which takes morphological data and information about the architecture of C. elegans neural network as an input, takes into account a set of parameters describing the models of muscular and nervous cells mentioned above, and automatically generates a software code written in NEURON's built-in programming language. The resulting code represents a model of electrophysiological activity and interaction between the following components, for which there is an experimental evidence that they are enough for producing backwards crawling movement [5]. It includes the majority of 95 body 
wall muscles (except those which are located in the head and are driven by other mechanisms) and 21 A-class motorneurons (VA1...VA12 and DA1...DA9, innervating ventral and dorsal muscles, correspondingly), which are distributed nearly uniformly from head to tail. In more details this system is described in our recent work [10].

What is currently included into the simulation:

1) The majority of 95 body wall muscles, excluding 6 of 23 or 24 cells in each of 4 muscle quadrants, for which we reproduce their simple geometry and location within the body

2) Motorneurons DA1...DA9 and VA1...VA12 with stretchreceptive processes.

3) Muscle arms from dBWML/dBWMR body wall muscles to DA- neurons.

4) Synaptic connections between muscle arms and DAneurons, connected via "Exp2Syn" NEURON's synapse model.

5) Synchronization between each pair of adjacent i-th and (i+1)-th dBWML muscle cells via gap junctions

6) Induced random activity of DA neurons, imitating their intrinsic rhythmic activity with typical frequencies.

\section{ACKNOWLEDGMENT}

Supported by the Russian Federation for Basic Research grant No. 18-07-00903.

\section{REFERENCES}

[1] Global brain dynamics embed the motor command sequence of Caenorhabditis elegans. Kato S, Kaplan HS, Schrödel T, Skora S, Lindsay TH, Yemini E, Lockery S, Zimmer M. Cell. 2015 Oct 22;163(3):656-69

[2] A. Palyanov, S. Khayrulin, S.D. Larson. "Three-dimensional realistic model of the Caenorhabditis elegans body and muscle cells in liquid and gel environments for behavioral analysis", Phil Trans $R$ Soc B: Biol Sci, 373(1758): 20170376, 2018.

[3] W.N. Nickell, R.Y.K. Pun, C.I. Bargmann, C.I. Kleene. "Single ionic channels of two Caenorhabditis elegans chemosensory neurons in native membrane", J. Membrane Biol., 189:55-66, 2002.

[4] A.Yu. Palyanov, A.S. Ratushnyak. "Some Details of Signal Propagation in the Nervous System of C. elegans", Russian Journal of Genetics: Applied Research, 5(6): 642-649, 2015.

[5] S. Gao, S.A. Guan, A.D. Fouad et al. "Excitatory motor neurons are local oscillators for backward locomotion", eLife, 7:e29915, 2018.

[6] S. Gao, M. Zhen. "Action potentials drive body wall muscle contractions in Caenorhabditis elegans". Proc Natl Acad Sci USA, 108(6): 2557-2562, 2011.

[7] P. Liu, D. Chen, Z.-W. Wang. "Postsynaptic current bursts instruct action potential firing at a graded synapse", Nature Communications, 4(1911), 2013.

[8] P. Liu, D. Chen, Z.-W. Wang. "Gap junctions synchronize action potentials and $\mathrm{Ca} 2+$ transients in Caenorhabditis elegans body wall muscle", J. Biol. Chem., 286(51):44285-93, 2011.

[9] J.G. White, E. Southgate, J.N. Thomson, S. Brenner. "The structure of the nervous system of the nematode Caenorhabditis elegans", Philos Trans R Soc Lond B Biol Sci, 314(1165):1-340, 1986.

[10] A.Yu. Palyanov and N.V. Palyanova. "On Prerequisites for Revealing C. elegans Backward Crawling Mechanism through Computer Simulation of Key Involved Subsystems", International MultiConference on Engineering, Computer and Information Sciences (SIBIRCON): 0944-0949, 2019. 